\title{
APLICABILIDADE DE INTERVENÇÕES PRIORITÁRIAS DA NIC PARA O DIAGNÓSTICO DE ENFERMAGEM DESOBSTRUÇÃO INEFICAZ DE VIASAÉREAS
}

\author{
A namaria A Ives N apoleãoํㅜㄹ Emília Campos de Carvalho²
}

RESUMO: 0 objetivo deste estudo foi identificar a aplicabilidade de intervenções prioritárias da Classificação das Intervenções de Enfermagem (NIC) para o diagnóstico de enfermagem Desobstrução ineficaz de vias aéreas em um centro de terapia intensiva pediátrico (CTIP) onde se adotam o processo de enfermagem e os diagnósticos de enfermagem da NANDA . 0 método utilizado foi o estudo de caso. Os enfermeiros listaram espontaneamente as atividades que desenvol viam e anal isaram, nas intervenções da NIC, se real izavam ou não as atividades adicionais que esta taxonomia apresenta. Identificou-se que eram realizadas no CTIP $84,6 \%$ das atividades da intervenção M onitorização Respiratória, $80,0 \%$ das atividades da intervenção Controle de Vias A éreas e $36,4 \%$ das atividades da intervenção Incremento da Tosse. Concluiu-se que a maioria das atividades das intervenções estudadas é realizada no CTIP e que a NIC pode oportunizar aos enfermeiros a identificação de potencialidades, necessidades e contribuir para melhorias na qualidade do cuidado prestado.

DESCRITORES: Enfermagem; Classificação; Diagnóstico de enfermagem; Cuidados de enfermagem.

\section{APPLICABILITY OF NIC PRIORITY INTERVENTIONS FOR THE NURSING DIAGNOSIS INFFECTIVE AIRWAY CLARANCE}

ABSTRACT: This study aimed to identify the applicability of NIC priority interventions to the diagnosis ineffectiveA irway clearance in a pediatric intensive care unit (PICU ), where the nursing process and NANDA's nursing diagnoses are adopted. The method used was the case study. The nurses spontaneously listed the activities they developed and analyzed whether they performed additional activities according to NIC taxonomy. The authors observed that in the PICU were performed $84.6 \%$ of the Respiratory M onitoring activities, $80.0 \%$ of the A irway $M$ anagement intervention activities and $36.4 \%$ of the activities regarding Cough Enhancement intervention. The conclusion was that the majority of the interventions studied are performed in the PICU and that NIC can give nurses the opportunity to identify potentialities and needs as well as to improve the quality of the care delivered.

DESCRIPTORS: N ursing; Classification; N ursing diagnosis; N ursing care.

\section{APLICABILIDAD DE INTERVENCIONES PRIORITARIAS DE NIC PARA EL DIAGNÓSTICO DE ENFERMERIA DESOBSTRUCCIÓN INEFICAZ DE VÍAS AÉREAS}

RESUMEN: El objetivo de este estudio fue identificar la aplicabilidad de intervenciones prioritarias de la Clasificación de las Intervenciones de Enfermería (NIC) para el diagnóstico de enfermería desobstrución ineficaz de vías aéreas en un centro de terapia intensiva pediátrico (CTIP) donde se adotpan el proceso de enfermería y los diagnósticos de enfermería de la NA NDA. EI método utilizado fue el estudio de caso. Los enfermeros alistaron espontáneamente las actividades que desarrollaron y analizaron, en las intervenciones de la NIC, si realizaban o no las actividades adicional es que esta taxonomía presenta. Se identificó que eran real izadas en CTIP 84,6\% de las actividades de la intervención M onitorización Respiratoria; $80,0 \%$ de las actividades de la intervencion Control de V ías A éreas; y 36,4\% de las actividades de la intervención Incremento da Tos. Se concluyó que la maioría de las actividades de las intervenciones estudiadas es realizada en CTIP y que la N IC puede dar oportunidad a los enfermeros de identificación de potencial idades, necesidades y contribuir para mejorías en la cualidade del cuidado prestado.

DESCRIPTORES: Enfermería; Clasificación; Diagnóstico de enfermería; Cuidados de enfermería.

\footnotetext{
${ }^{1}$ Professora adjunto do Departamento de Enfermagem da Universidade Federal de São Carlos - UFSCar. Doutora em Enfermagem pelo programa Interunidades de Doutoramento - Escola de Enfermagem de Ribeirão Preto da Universidade de São Paulo - EERP/USP.

2 Professora titular da EERP/USP
} 


\section{INTRODUÇÃO}

0 estudo das intervenções de enfermagem teve como seu principal foco de interesse o comportamento do enfermeiro, bem como suas ações para auxiliar 0 paciente a alcançar o resultado desejado ${ }^{(1)}$. N este sentido a Classificação das Intervenções de Enfermagem (NIC) é reconhecida por sua importância no sentido de nomear as ações real izadas pela equipe de enfermagem.

A lgumas das razões para o desenvolvimento da NIC foram a padronização da nomenclatura dos tratamentos de enfermagem, a expansão do conhecimento de enfermagem sobre as ligações entre diagnósticos, tratamentos e resultados, 0 desenvolvimento de sistemas de informação de enfermagem, 0 ensino do processo de tomada de decisão para al unos de enfermagem, a determinação dos custos dos serviços realizados por enfermeiras, o planejamento dos recursos necessários nos locais da prática de enfermagem e a linguagem única para comunicar a função da enfermagem ${ }^{(2)}$.

Considera-se de grande valor a utilização de taxonomias ou sistemas de classificação na prática de enfermagem. Em se tratando de uma taxonomia de intervenções, esse valor pode se concretizar na possibilidade de facilitar a construção de planos de cuidados de enfermagem, de nomear as ações realizadas, de facilitar o registro dessas ações, de reforçar a importância de ações efetivamente realizadas no cotidiano, de conferir maior visibilidade ao trabal ho do enfermeiro e ainda de identificar atividades não realizadas e discutir os aspectos relacionados à sua não realização, como os legais, estruturais, condições de trabal ho, entre outros.

Entende-se que a utilização de uma taxonomia requer a compreensão de suas particularidades conceituais e estruturais. Em relação à NIC, os enfermeiros que desejam utilizá-la devem se familiarizar com sua estrutura taxonômica e com a definição de termos apresentados, como, por exemplo, os termos "intervenção" e "atividade", que comumente são utilizados sem possuírem, necessariamente, uma relação entre si e na NIC possuem especificidade e interrelacionamento.

0 termo "intervenção de enfermagem" édefinido na NIC como "qualquer tratamento baseado no julgamento e conhecimento clínicos, realizado por um enfermeiro para aumentar os resultados do paciente/ cliente" (2:xxi). U ma intervenção é composta por uma série de atividades.

As atividades são definidas como "(...) ações específicas realizadas por enfermeiros para implementar uma intervenção (...). As atividades situam-se no nível concreto da ação. Há necessidade de uma série de atividades para implementar uma intervenção"(2:xxii).

Em suas publicações mais recentes encontra-se disponível na NIC um capítulo que apresenta os diagnósticos de enfermagem da NANDA e as intervenções que podem ser apropriadas a cada um deles. Nele, as intervenções são apresentadas em três níveis: primeiro nível ou intervenções prioritárias, que são intervenções mais prováveis para a resolução daquele diagnóstico; segundo nível ou intervenções sugeridas: intervenções dirigidas ao diagnóstico, mas não como uma intervenção prioritária para a maioria dos pacientes com aquele diagnóstico e terceiro nível ou intervenções adicionais optativas: intervenções aplicadas somente para alguns pacientes com o diagnóstico(2).

É inegável a magnitude do trabalho de organizar as intervenções de enfermagem em uma estrutura taxonômica. A ssim, face às características até aqui apontadas sobre a NIC, considera-se importante 0 desenvolvimento de estudos acerca desta taxonomia e do quanto suas intervenções são aplicáveis à realidade brasileira.

Julgou-se, então, pertinente o desenvolvimento de um estudo sobre a aplicabilidade da NIC em uma unidade hospital ar onde o cuidado de enfermagem ocorre de forma sistematizada, partindo-se de um diagnóstico deenfermagem da NANDA que supostamente constituía resposta humana freqüentemente identificada entre os pacientes no local do estudo.

Desta forma, optou-se pela realização desteestudo em uma U nidade de Terapia Intensiva Pediátrica de um hospital escola que possuía as características acima citadas e pela escolha do diagnóstico de enfermagem da NANDA Desobstrução ineficaz de vias aéreas ${ }^{(3)}$ visto que, além de constituir resposta humana freqüentemente citada na literatura de enfermagem em diferentes situações e contextos(4-8), constava em impresso específico de diagnósticos de enfermagem da referida unidade. O ptou-seainda pela escolha do fator relacionado Presença de via aérea artificial, uma vez que, conforme é apontado na literatura, todos os pacientes que possuem uma via aérea artificial apresentam-se com risco de desenvolver retenção das secreções ${ }^{(4,9)}$.

A demais, esse fator relacionado confere aos pacientes uma maior complexidade clínica, o que responsabiliza legalmente 0 enfermeiro pela tomada de decisões em relação aos mesmos, conforme consta na legislação brasileira, Decreto n-94.406, de 08 de junho

Cogitare Enferm 2007 jan/mar; 12(1):9-19 
de 1987, que regulamenta a Lei $n-7.498$, de 25 de junho de 1986. Em seu artigo 80, itens g e $h$ consta que, ao enfermeiro incumbe privativamente "cuidados diretos de Enfermagem a pacientes graves com risco de vida" e "cuidados de enfermagem de maior complexidade técnica e que exijam conhecimentos científicos adequados e capacidade de tomar decisões imediatas" (10).

Diante do exposto, o presente estudo teve como objetivo identificar a aplicabilidade de intervenções prioritárias da NIC para o diagnóstico de enfermagem Desobstrução ineficaz de vias aéreas relacionada à presença de via aérea artificial em um Centro de Terapia Intensiva Pediátrico (CTIP) do estado de São Paulo.

\section{METODOLOGIA}

Trata-se de estudo de caso realizado no CTIP de um hospital escola de alta complexidade. A opção pelo método de estudo de caso se deu uma vez que, pretendiase realizar uma abordagem centrada no CTIP escol hido, onde 0 processo de enfermagem e 0 uso da taxonomia da NA NDA já estavam implementados e também por entender que os resultados obtidos no presente estudo poderiam subsidiar hipóteses para a realização de trabal hos futuros, uma das características que podem ser consideradas para a escolha deste método ${ }^{(11)}$.

Este estudo foi preliminarmente submetido ao Comitê de Ética em Pesquisa com Seres Humanos da instituição hospitalar onde foi realizado, com aprovação. Todos os aspectos éticos para sua realização foram observados. A ntes do início da coleta de dados os objetivos da pesquisa foram explicitados pela autora, 0 Termo de Consentimento Livre e Esclarecido foi apresentado e a coleta somente era iniciada após a concordância em participar do estudo e a assinatura do termo pela enfermeira, que tinha assegurada, no conteúdo deste termo, a liberdade de deixar de participar do estudo a qual quer momento.

Participaram dele as sete enfermeiras atuantes no CITP, sendo que, na segunda etapa de análise (análise evocada), houve uma demissão. Quanto à idade, uma enfermeira apresentava mais de 40 anos, duas entre 30 a 35 anos, três entre 20 e 25 anos e uma menos de 25 anos. 0 tempo de atuação na enfermagem das enfermeiras variou de dois a 13 anos, todas atuando no mínimo há dois anos no setor.

Em relação à qualificação profissional, duas enfermeiras cursavam M estrado em Enfermagem, uma era especialista em enfermagem pediátrica, uma possuía especialização em outras áreas e duas possuíam somente graduação em enfermagem.

Foram utilizadas as intervenções apresentadas na NIC (2) como prioritárias ou de primeiro nível para o diagnóstico de enfermagem Desobstrução ineficaz de vias aéreas.

Buscou-se conhecer a aplicabilidade destas intervenções nas seguintes situações: a) espontaneamente, a partir do levantamento, junto às enfermeiras, das atividades que realizavam no CTIP no atendimento a crianças com o diagnóstico de enfermagem desobstrução ineficaz de vias aéreas relacionada à presença de via aérea artificial e b) por análise evocada, quando as atividades não mencionadas pelas enfermeiras espontaneamente, mas constantes nas intervenções da NIC para o diagnóstico de enfermagem Desobstrução ineficaz de vias aéreas, foram a elas apresentadas para análise quanto à sua realização no CTIP.

Na primeira situação foi utilizado um instrumento de coleta de dados no qual as enfermeiras listavam as atividades que realizavam no atendimento às crianças com o referido diagnóstico de enfermagem.

A s enfermeiras buscavam relembrar as ações que realizavam e consultavam a lista de intervenções de enfermagem impressa que utilizavam nesse setor para a prescrição de enfermagem. A s atividades foram listadas e posteriormente todas as enfermeiras tiveram acesso a este elenco de atividades.

As ações mencionadas espontaneamente pelas enfermeiras foram arroladas e comparadas às atividades contidas nas intervenções prioritárias da NIC para 0 diagnóstico de enfermagem Desobstrução ineficaz de vias aéreas por meio de mapeamento comparativo, conforme estudo que apresenta proposta de regras para mapeamento das intervenções de enfermagem constantes de um sistema informatizado de um hospital às intervenções da $\mathrm{NIC}^{(12)}$. As regras apresentadas neste estudo são: 1- proceder do título das intervenções da NIC para as atividades da NIC; 2- usar o contexto dos diagnósticos de enfermagem; 3- mapear significados versus palavras; 4- usar a intervenção mais específica da NIC.

No presente estudo, foi identificada maior facilidade em desenvolver o mapeamento utilizando a segunda regra em primeiro lugar e a primeira regra em segundo lugar.

0 mapeamento foi avaliado por cinco enfermeiras, sendo duas doutoras em enfermagem e três mestres em enfermagem, com no mínimo cinco anos de atuação no ensino ou na assistência de enfermagem, que atuavam 
utilizando o processo de enfermagem e os diagnósticos de enfermagem da N A NDA . Houve pequenas sugestões por parte das enfermeiras avaliadoras de se considerar atividades mais específicas apontadas pelas enfermeiras do CTIP como correspondentes a atividades da NIC mais gerais. As autoras discutiram as sugestões apontadas e as mesmas foram acatadas por sua coerência.

Como resultado do mapeamento obteve-se uma série de atividades contidas na NIC e que não haviam sido mencionadas espontaneamente pelas enfermeiras como realizadas no CTIP.

Partindo-se destas atividades, passou-se, então, para a identificação da aplicabilidade por meio de análise evocada, ou seja, as atividades constantes na NIC e não mencionadas espontaneamente pelas enfermeiras foram a elas apresentadas para análise quanto à sua realização no CTIP.

Nesta etapa, cada intervenção prioritária foi apresentada na íntegra, ou seja, as atividades que já haviam sido mencionadas pelas enfermeiras na primeira etapa constavam e apenas foram destacadas, não sendo necessário às enfermeiras analisá-las. Todas as enfermeiras concordaram com as atividades listadas na primeira etapa e mapeadas com as atividades das intervenções da NIC.

Estabeleceu-se que, caso houvesse divergências entre as enfermeiras em relação à informação sobre a real ização ou não das atividades na análise evocada, isto seria discutido considerando-se estas divergências, buscando compreender os fatores que pudessem estar associados a elas. No entanto, para a grande maioria das atividades estudadas nesta situação (76,3\%), observouse uma concordância entre todas as enfermeiras em relação à real ização ou não das mesmas, sendo que, para as demais atividades $(23,7 \%)$, apenas uma enfermeira apresentou respostas diferentes. Sendo assim, estas atividades foram consideradas realizadas ou não realizadas de acordo com o que foi mencionado pela maioria das enfermeiras.

\section{RESULTADOS}

Três são as intervenções prioritárias apresentadas na ligação dos diagnósticos de enfermagem da NA NDA com as intervenções da NIC para o diagnóstico de enfermagem Desobstrução ineficaz de vias aéreas: controle de vias aéreas, incremento da tosse e monitorização respiratória(2).

Na primeira situação, em que se buscou levantar espontaneamente entre as enfermeiras as ações que real izavam no atendimento a crianças com o diagnóstico de enfermagem Desobstrução ineficaz de vias aéreas e compará-las por meio de mapeamento às intervenções prioritárias da NIC para este diagnóstico, constatou-se que as atividades listadas pelas enfermeiras possuíam correspondência com apenas 19 (33,3\%) das 57 (100,0\%) atividades que compunham estas intervenções.

N a segunda situação, ou seja, na análise evocada, em que as atividades que compunham estas intervenções prioritárias e que não foram mencionadas pelas enfermeiras espontaneamente foram apresentadas às mesmas para análise quanto à sua realização no CTIP, constatou-se que, embora não mencionadas pelas enfermeiras espontaneamente, estas atividades foram consideradas, em sua maioria, como sendo realizadas no CTIP.

Os dados referentes às atividades anal isadas pelas enfermeiras na primeira e na segunda situação estão apresentados na Tabela 1.

Tabela 1 - A plicabilidade das intervenções prioritárias apresentadas na NIC (2) para o diagnóstico de enfermagem Desobstrução ineficaz de vias aéreas referida pelas enfermeiras do CTIP espontaneamente (antes da análise das intervenções da N IC) e por análise evocada (após análise das intervenções da NIC). Ribeirão Preto, 2004

\begin{tabular}{lcccccccc}
\hline Intervenções & $\begin{array}{c}\text { Atividades } \\
\text { realizadas - } \\
\text { referidas } \\
\text { espontaneamente }\end{array}$ & $\begin{array}{c}\text { Atividades } \\
\text { realizadas - } \\
\text { análise evocada }\end{array}$ & $\begin{array}{c}\text { Atividades não } \\
\text { realizadas - } \\
\text { análise evocada }\end{array}$ & Total \\
\hline Monitorização respiratória & $\mathrm{N}$ & $\%$ & $\mathrm{~N}$ & $\%$ & $\mathrm{~N}$ & $\%$ & $\mathrm{~N}$ & $\%$ \\
Controle de vias aéreas & 11 & 42,3 & 11 & 42,3 & 4 & 15,4 & 26 & 100,0 \\
Incremento da tosse & 8 & 40,0 & 8 & 40,0 & 4 & 20,0 & 20 & 100,0 \\
Total & - & - & 4 & 36,4 & 7 & 63,6 & 11 & 100,0 \\
\hline
\end{tabular}

Cogitare Enferm 2007 jan/mar; 12(1):9-19 
Em relação à análise por intervenção, a intervenção M onitorização Respiratória apresentou 0 maior número de atividades consideradas realizadas (84,6\%). Estas atividades estão descritas no Quadro 1.

Quadro 1 - A tividades da intervenção M onitorização Respiratória realizadas no CTIP. Ribeirão Preto, 2004

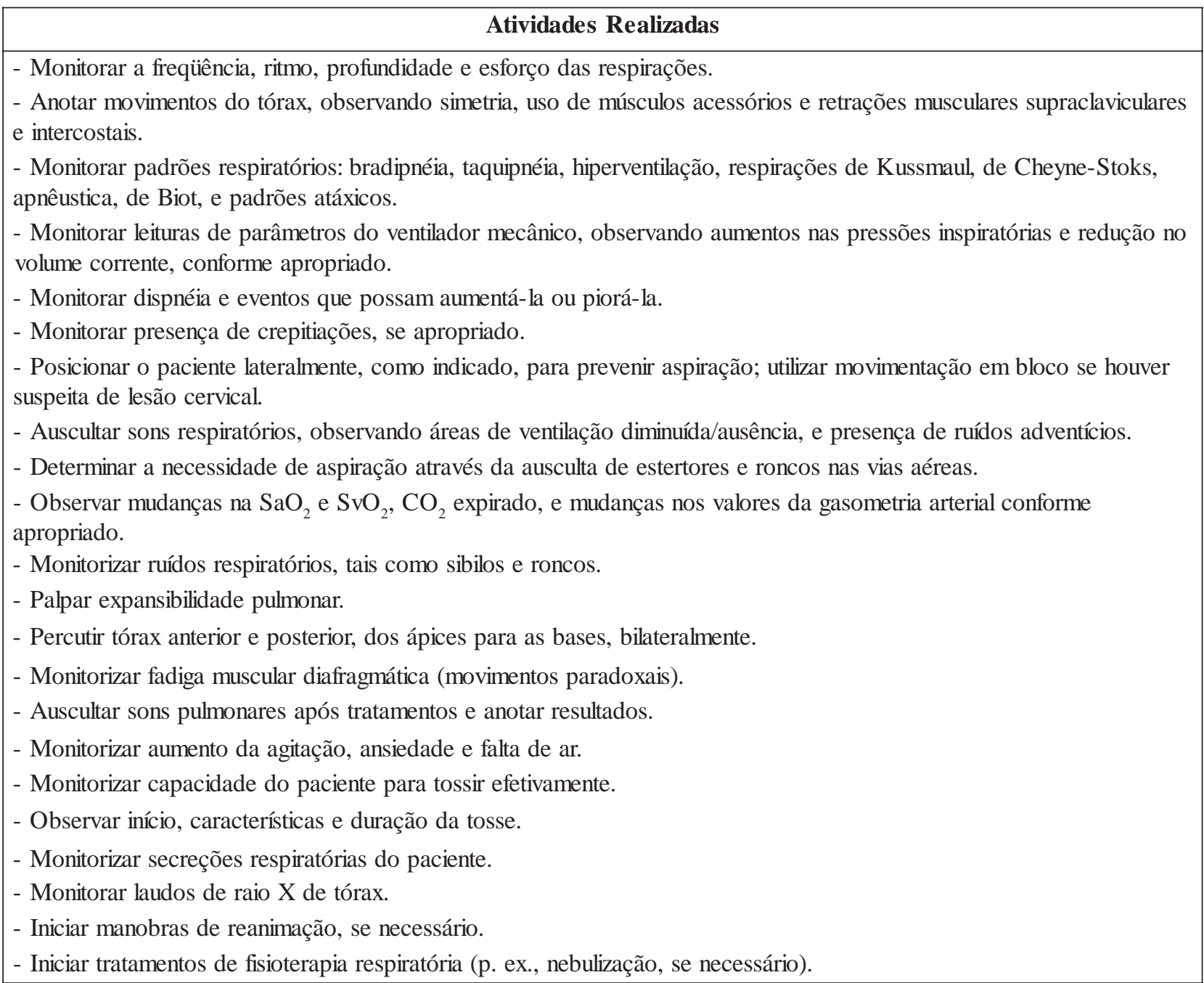

As atividades da intervenção M onitorização Respiratória apontadas como não realizadas (15,4\%) estão descritas no Quadro 2. À frente das atividades constam justificativas gerais apontadas para sua não realização no CTIP.

Quadro 2 - A tividades da intervenção M onitorização Respiratória não realizadas no CTIP e justificativas atribuídas pelas enfermeiras para sua não real ização. Ribeirão Preto, 2004

\begin{tabular}{|c|c|}
\hline Atividades não realizadas & Justificativas \\
\hline $\begin{array}{l}\text { - Observar localização da traquéia. } \\
\text { - Monitorizar valores das provas de função pulmonar, } \\
\text { capacidade vital, força inspiratória máxima, volume } \\
\text { expiratório forçado em um segundo, volume expiratório, } \\
\text { forçado/capacidade vital forçada, conforme disponibilidade. } \\
\text { - Monitorizar rouquidão na voz, de hora em hora, em } \\
\text { pacientes com queimaduras faciais. } \\
\text { - Abrir vias aéreas usando a técnica da elevação do } \\
\text { queixo, ou da mandibula, como apropriado. }\end{array}$ & $\begin{array}{l}\text { - Procedimento não realizados habitualmente no CITP. } \\
\text { - Falta de tempo. } \\
\text { - Falta de conhecimento. } \\
\text { - Não adequado às condições clínicas dos pacientes do } \\
\text { CTIP, na maioria das vezes. }\end{array}$ \\
\hline
\end{tabular}


A intervenção Controle de Vias A éreas consideradas realizadas pelas enfermeiras (80,0\%). apresentou 0 segundo maior número de atividades Estas atividades estão apresentadas no Quadro 3.

Quadro 3 - A tividades da intervenção Controle de Vias A éreas realizadas no CTIP. Ribeirão Preto, 2004

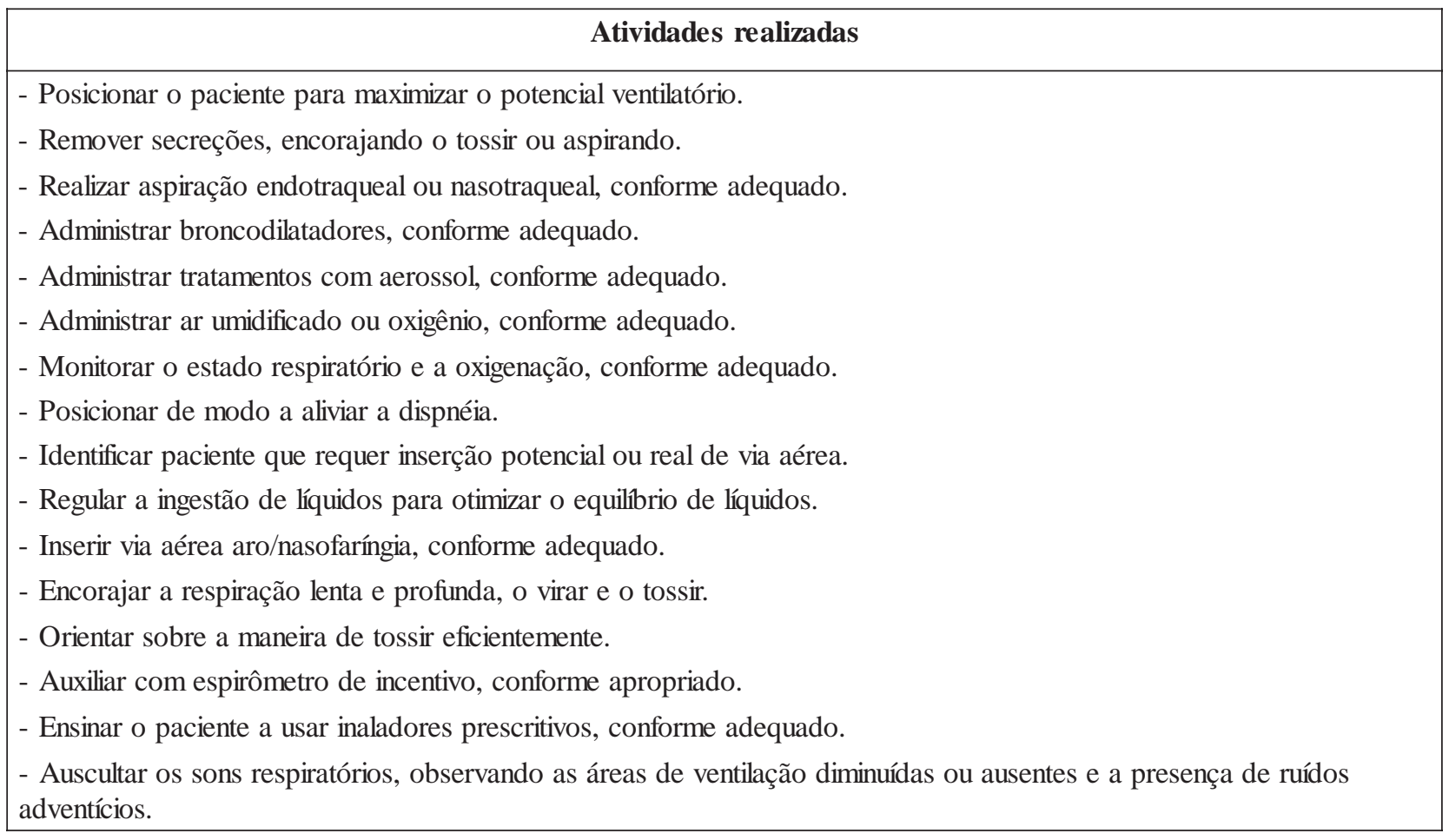

As atividades da intervenção Controle de Vias justificativas gerais apontadas pelas enfermeiras para sua A éreas apontadas como não realizadas $(20,0 \%)$ e não realização no CTIP estão descritas no Quadro 4.

Quadro 4 - A tividades da intervenção Controle de Vias A éreas não realizadas no CTIP ejustificativas atribuídas pelas enfermeiras à sua não realização. R ibei rão Preto, 2004

\begin{tabular}{|l|l|}
\hline \multicolumn{1}{|c|}{ Atividades não realizadas } & \multicolumn{1}{c|}{ Justificativas } \\
\hline - Realizar fisioterapia do tórax, conforme adequado. & - Não está de acordo com a legislação brasileira. \\
- Administrar tratamentos com nebulizador ultra-sônico, & - Recurso não disponível no serviço. \\
conforme adequado. & - Não condizente com a condição clínica dos pacientes, na \\
- Abrir a via aérea usando a técnica de elevação do queixo & maioria das vezes. \\
ou manobra de elevação da mandíbula, conforme & - Falta de tempo. \\
adequado. & - Não incorporação da atividade por parte da equipe do \\
- Usar técnicas lúdicas para encorajar a respiração & CTIP. \\
profunda de crianças (por exemplo, soprar bolhas com \\
instrumento específico; soprar catavento, apito, gaita de \\
boca, balões, língua-de-gato; realizar competição de sopro \\
usando bolas de pingue-pongue, etc.).
\end{tabular}

A intervenção Incremento da Tosse apresentou um número menor de atividades consideradas realizadas $(36,4 \%)$ em relação às não realizadas $(63,6 \%)$. De $11(100,0 \%)$ atividades que compõem esta intervenção, $4(36,4 \%)$ foram consideradas realizadas e $7(63,6 \%)$ não realizadas. Todas as atividades desta intervenção foram submetidas à análise evocada, uma vez que não houve atividades correspondentes mencionadas espontaneamente pelas enfermeiras. 
As atividades da intervenção Incremento da estão descritas no Quadro 5. Tosse, apontadas pelas enfermeiras como realizadas,

Quadro 5 - A tividades da intervenção Incremento da Tosse realizadas no CTIP. Ribeirão Preto, 2004

\begin{tabular}{|l|}
\hline \multicolumn{1}{|c|}{ Atividades realizadas } \\
\hline Auxiliar o paciente a sentar-se com a cabeça levemente fletida, os ombros relaxados e os joelhos flexionados. \\
Encorajar o paciente a fazer várias respirações profundas. \\
Encorajar o uso de espirometria de incentivo, conforme apropriado. \\
Promover a hidratação sistêmica com líquidos, conforme apropriado. \\
\hline
\end{tabular}

As atividades da intervenção Incremento da tosse apontadas pelas enfermeiras como não realizadas estão descritas no Q uadro 6, assim como justificativas gerais apontadas para sua não real ização.

Quadro 6 - A tividades da intervenção Incremento da Tosse não realizadas no CTIP e justificativas atribuídas à não real ização. R ibeirão Preto, 2004

\begin{tabular}{|l|l|}
\hline \multicolumn{1}{|c|}{ Atividades } & \multicolumn{1}{|c|}{ Justificativas } \\
\hline - Monitorar os resultados dos testes de função pulmonar, & - Procedimentos não são realizados habitualmente no \\
especialmente a capacidade vital, a força inspiratória máxima, o & CTIP. \\
volume expiratório forçado em um segundo (VEF) e EVF/CVF, & - Atividade desempenhada pelo fisioterapeuta. \\
conforme apropriado. & - Não há consenso sobre a realização da atividade no \\
- Encorajar o paciente a fazer uma respiração profunda, segurar & CTIP. \\
o ar por dois segundos e tossir duas ou três vezes sucessivas. & \\
- Orientar o paciente a inalar profundamente várias vezes, & \\
expirar lentamente para frente e provocar 3 ou 4 acessos (contra & \\
a glote aberta). & \\
- Orientar o paciente a inalar profundamente várias vezes, & \\
expirar lentamente e tossir ao final da expiração. & \\
- Iniciar técnicas de compressão da parede lateral do tórax & \\
durante a fase de expiração da manobra de tosse, conforme & \\
apropriado. & \\
- À medida que o paciente tosse, repentinamente comprimir o & \\
abdômen abaixo do xifóide com a mão espalmada, enquanto & \\
auxilia o paciente a flexionar-se para frente. & \\
- Orientar o paciente no sentido de seguir tossindo com várias & \\
respirações de inalação máxima. & \\
\hline
\end{tabular}

\section{DISCUSSÃO}

Observou-se que várias atividades não mencionadas espontaneamente pelas enfermeiras foram identificadas na NIC como sendo real izadas no atendimento a crianças com o diagnóstico de enfermagem Desobstrução ineficaz de vias aéreas. Como as enfermeiras utilizaram o impresso de prescrição de enfermagem existente para ajudaremnas a lembrar das ações que real izavam, supõe-se que muitas destas atividades podem ser realizadas sem 0 devido registro no cotidiano do CTIP.

Em estudo realizado em um hospital brasileiro sobre intervenções de enfermagem para pacientes com Desobstrução ineficaz de vias aéreas, foram examinadas as prescrições de enfermagem nos prontuários e observado que somente as ações já padronizadas no local eram prescritas para os pacientes, ou seja, nenhuma outra ação específica foi registrada. Tal fato levou as autoras à suposição de que nem tudo o que foi realizado foi registrado ${ }^{(13)}$.

Diante do exposto, entende-se que a utilização 
da NIC pode constituir elemento auxiliar na identificação de ações efetivamente realizadas pela equipe de enfermagem e que não são registradas de alguma forma.

Outro aspecto importante a ser considerado refere-se à autonomia dos enfermeiros para desenvolverem as atividades constantes nas intervenções da NIC, isto é, que ações seriam dependentes de prescrições médicas e que ações seriam independentes de prescrição médica.

Para tanto, adotou-se a denominação utilizada em estudo brasileiro sobre a NIC em que as autoras identificaram as atividades da intervenção Controle de líquidos que eram realizadas e consideradas importantes pelas enfermeiras em um hospital especializado. A s autoras identificaram que a maioria das atividades desta intervenção foi considerada sempre realizada e sempre importante. A s atividades foram classificadas como "dependentes" e "independentes", de acordo com a necessidade de solicitação médica para a realização das mesmas e observou-se que, para um grupo específico de atividades em que houve concordância total entre as enfermeiras quanto à realização e importância das mesmas, houve predominância de atividades dependentes de prescrição médica ${ }^{(14)}$.

No presente estudo, as intervenções M onitorização respiratória e Controle de vias aéreas tiveram a maioria de suas atividades consideradas realizadas no CTIP, ao passo que a intervenção Incremento da tosse apresentou um número menor de atividades consideradas realizadas em relação às consideradas não realizadas. Este fato chama a atenção, uma vez que esta intervenção é apresentada na NIC como prioritária no tratamento de pacientes com o diagnóstico de enfermagem Desobstrução ineficaz de vias aéreas.

A ssim, pode-se sugerir que as intervenções apresentadas pela NIC oferecem aos enfermeiros a possibilidade de identificação de ações específicas para determinado diagnóstico de enfermagem que podem passar a fazer parte dos planos de cuidados dos pacientes. Neste caso, a idéia de aplicabilidade não se limitaria ao que efetivamente se realiza, mas ao que é passível de ser realizado.

Entre as atividades da intervenção M onitorização respiratória realizadas no CTIP, observa-se que grande parte é referente ao exame físico e ao controle de parâmetros mais específicos. Em menor número são encontradas ações a serem realizadas junto ao paciente para melhorar seu estado respiratório.

Entre as atividades desta intervenção não realizadas no CTIP, encontram-se aquelas mais específicas a certos grupos de pacientes, como os que apresentam queimaduras e que requerem cuidados emergenciais, além de atividade de acompanhamento de valores de exames de prova de função pulmonar.

As enfermeiras apontaram, entre as justificativas para a não realização destas atividades, dificuldades de implementação como, fal ta de tempo e de conhecimento, além de sua não adequação às condições clínicas do paciente do CTIP, na maioria das vezes.

Nesse sentido, entende-se que os enfermeiros, ao usar uma taxonomia de intervenções, podem se sentir estimulados a refletir acerca de atividades que não estão sendo implementadas e levantar necessidades da equipe de enfermagem em relação ao seu preparo para o desenvolvimento de determinadas atividades. $A$ menção à falta de tempo chama a atenção para a possibilidade de que necessidades relativas às condições de trabalho possam igualmente ser levantadas quando se identificam outras possibilidades de atuação que acarretariam melhor qualidade do cuidado prestado.

A inda, entre as atividades da intervenção M onitorização respiratória consideradas realizadas no CTIP, identifica-se uma predominância daquel as que, supostamente, podem ser iniciadas pelo enfermeiro independente de prescrição médica, tais como, "monitorizar padrões respiratórios: bradipnéia, taqui pnéia, hiperventilação, respirações de K ussmaul, de Cheyne-Stokes, apnêustica, de Biot, e padrões atáxicos", "monitorizar leituras de parâmetros do ventilador mecânico, observando aumentos nas pressões inspiratórias e redução no volume corrente, conforme apropriado", "monitorar dispnéia e eventos que possam aumentá-la ou piorá-la", "auscultar sons respiratórios, observando áreas de ventilação diminuídas/ausentes, e presença de ruídos adventícios".

A ssim, as atividades desta intervenção da NIC, além de possuírem aplicabilidade pela equipe de enfermagem, evidenciam a autonomia do enfermeiro e seu importante papel para a resolubilidade do diagnóstico de enfermagem D esobstrução ineficaz de vias aéreas. Destaca-se ainda, a importância do registro destas atividades no cotidiano, no sentido de fornecer dados para a efetividade do cuidado prestado, conferir maior visibilidade em relação às atividades 
realizadas pelos enfermeiros, para fins legais caso se faça necessário e, ainda, para prover dados relativos ao trabalho dos enfermeiros para pesquisa, ensino, entre outros.

$E m$ relação às atividades da intervenção Controle de vias aéreas consideradas realizadas no CTIP, observa-se que a maioria corresponde a ações junto ao paciente para melhora do seu estado respiratório, tais como, posicioná-lo de modo a al iviar a dispnéia, realizar aspiração endo ou nasotraqueal, entre outras. Tal como em relação às atividades da intervenção M onitorização respiratória, identifica-se que, supostamente, a maioria se refere a atividades independentes, ou seja, que podem ser iniciadas pelo enfermeiro, com exceção da atividade "regular a ingestão de líquidos para otimizar o equilíbrio de líquidos".

Entre as atividades da intervenção Controle de vias aéreas não realizadas no CTIP, foram identificadas atividades realizadas junto ao paciente para melhora de seu estado respiratório. A o justificar a não realização das atividades, a "fisioterapia do tórax" foi apontada como atividade que está, supostamente, em desacordo com a legislação brasileira. Em consulta à legislação específica da Enfermagem, não há menção de impedimento ao enfermeiro em realizar atividades de fisioterapia do tórax ${ }^{(10)}$.

$\mathrm{Na}$ literatura de enfermagem, identifica-se a fisioterapia torácica como intervenção que promove a mobilização de secreções pul monares. São descritas a drenagem postural, percussão torácica e vibração. Os autores chamam a atenção para a necessidade de conhecimento na indicação da fisioterapia torácica sendo que, alguns enfocam também os riscos existentes a partir do uso desta terapêutica ${ }^{(15-19)}$. O utros autores apontam a fisioterapia torácica como intervenção colaborativa, que, conforme descrevem, refere-se a intervenção iniciada/realizada em conjunto com outros profissionais ${ }^{(20)}$.

N o entanto, a não realização desta atividade no CTIP reflete a complexidade da questão de realização de atividades de fisioterapia pela equipe de enfermagem, aparentemente pouco discutida e elucidada, sendo necessário reflexão e tomada de decisão buscando assegurar que o paciente receba todos os cuidados necessários à sua recuperação. Vale lembrar que a NIC é apontada como um facilitador da colaboração multidisciplinar, uma vez que provêuma linguagem padronizada, passível de entendimento por enfermeiras de diferentes especialidades assim como a profissionais de outras disciplinas ${ }^{(21)}$.

Em relação à atividade não realizada que envolve o lúdico, a maior complexidade freqüentemente observada nos pacientes nas unidades de terapia intensiva pode também contribuir para a não realização deste tipo de atividade. A "não incorporação da atividade por parte da equipe", justificativa mencionada, pode refletir a dificuldade dos profissionais destas unidades em exercerem atividades que demandam maior tempo junto à criança e também a participação da mesma.

Nesse sentido, mais uma vez, considera-se que a utilização de uma taxonomia de intervenções pode despertar questionamentos entre os profissionais e suscitar discussões e reflexões que, de al guma forma, promovam mel hora na qual idade do cuidado prestado e, conseqüentemente, favoreçam o alcance dos resultados junto ao paciente.

Em relação à intervenção Incremento da tosse, observa-se que, entre as quatro atividades realizadas no CTIP, todas são de realização direta junto ao paciente, complementares para a melhoria de seu estado respiratório, sendo que, supostamente, apenas uma atividade pode ser entendida como dependente de prescrição médica para sua realização, qual seja, "promover a hidratação sistêmica". Em relação ao encorajamento para a realização de espirometria de incentivo, a maioria das enfermeiras apontou que se trata de atividade realizada quando esta terapêutica foi indicada para a criança pelo fisioterapeuta.

Entre as sete atividades da intervenção Incremento da tosse não realizadas no CTIP observase, principalmente, atividades relacionadas ao ensino da tosse. A justificativa apontada para a não realização destas atividades no CTIP foi a de que são realizadas pelo fisioterapeuta. Contudo, o ensino da tosse é também uma atividade preconizada na literatura da enfermagem e sua eficácia é enfatizada pelos mesmos nos casos de presença de secreção nas vias aéreas ${ }^{(15-18)}$.

Embora se trate de uma intervenção prioritária da NIC nos casos em que se diagnostica Desobstrução ineficaz de vias aéreas nos pacientes, as atividades de ensino da tosse também são mais comumente direcionadas às crianças que não se encontram em estado grave e demandam a participação da criança e um maior tempo junto à mesma, o que pode contribuir para a sua não real ização pelo enfermeiro no CTIP.

Diante dos resultados obtidos, pode-se afirmar que os enfermeiros do CTIP realizam a maioria das 
atividades das intervenções apontadas na NIC como prioritárias na vigência de Desobstrução ineficaz de vias aéreas nos pacientes, indicando uma considerável aplicabilidade destas intervenções neste serviço.

Em relação às atividades que não são realizadas, evidenciou-se que, uma vez identificadas, os enfermeiros possuem a oportunidade de refletir sobre o cuidado que vem sendo prestado e as implicações da não realização das mesmas junto aos pacientes com o diagnóstico de enfermagem estudado, no que diz respeito ao alcance dos resultados esperados para os pacientes. 0 utros aspectos importantes para reflexão a partir da identificação de atividades não realizadas referem-se à necessidade de apropriação de determinados conhecimentos por parte da equipe de enfermagem, às condições de trabal ho que favorecem ou desfavorecem a sua realização, à necessidade e formas de integração da enfermagem numa perspectiva interdisciplinar, entre outras questões.

\section{CONSIDERAÇÕES FINAIS}

0 presente estudo possibilitou identificar a aplicabilidade de três intervenções da NIC em um CTIP brasileiro, a partir da identificação, pelos enfermeiros que nele atuam, de atividades real izadas e não realizadas em determinada situação, qual seja, quando se diagnostica D esobstrução ineficaz de vias aéreas relacionada à presença de via aérea artificial.

Pôde-se identificar que, entre as atividades da intervenção M onitorização respiratória, 84,6\% são real izadas no CTIP; em rel ação à intervenção Controle de vias aéreas, $80,0 \%$ das atividades foram consideradas realizadas no CTIP e em relação à intervenção Incremento da tosse, $36,4 \%$ das atividades foram consideradas realizadas.

Os resultados obtidos permitiram identificar as atividades ef etivamente real izadas pelos enfermeiros e constatar que a maioria dessas atividades supostamente pode ser realizada independente de prescrição médica.

Entre as atividades não realizadas no CTIP predominam aquelas relativas à fisioterapia respiratória e ao ensino da tosse, que, segundo foi apontado pelos enfermeiros, são realizadas pelo fisioterapeuta. Em relação a outras atividades não realizadas, os enfermeiros justificaram, de uma maneira geral, que não eram condizentes com a condição clínica dos pacientes do CTIP, tais como as manobras de abertura das vias aéreas, que nem sempre se aplicam na presença de vias aéreas artificiais.

0 estudo possibilitou verificar, ainda, que, ao apresentar as intervenções da NIC aos enfermeiros, os mesmos puderam identificar várias atividades que real izam cotidianamente no CTIP junto aos pacientes com o diagnóstico de enfermagem estudado, mas que não foram por eles citadas espontaneamente mesmo consultando o impresso de prescrição de enfermagem, 0 que sugere 0 sub-registro destas atividades cotidianamente.

Em relação às atividades não realizadas, os resultados chamam à reflexão acerca do espectro de atuação do enfermeiro, da possível fragmentação do cuidado prestado e da apropriação do conhecimento pela equipe de enfermagem. Outro aspecto importante refere-se à discussão acerca do quanto a incorporação destas atividades pela equipe de enfermagem auxiliaria o paciente a alcançar os resultados esperados.

Pelo grande número de intervenções e atividades apresentadas na NIC, pode-se inferir que sua utilização será facilitada com o uso de sistemas informatizados. A utores brasileiros apontam para a necessidade de estudos e testes de protocolos de intervenções de enfermagem a partir das ligações entre a taxonomia da NA NDA e as intervenções da NIC e de avaliação dos resultados utilizando a classificação de resultados de enfermagem - NOC (22).

Entende-se que é importante vislumbrar ferramentas que facilitem a utilização da NIC, assim como de outros sistemas de classificação que auxiliem os enfermeiros a otimizarem seu trabal ho na busca de melhorias da qualidade do cuidado prestado.

Os resultados aqui obtidos evidenciaram, ainda, que estudos de elementos da NIC, realizados junto a enfermeiros em unidades específicas de cuidados à saúde, podem trazer contribuições ao possibilitar a aproximação desses com a estrutura da taxonomia, suscitar questionamentos, reflexões, identificar aspectos para pesqui sas futuras e reforçar a relevância do trabalho de enfermagem nos diferentes contextos de cuidado à saúde.

\section{REFERÊNCIAS}

1. J ohnson M, Bulechek $G$, Dochterman JM, M aas $M$, M oorhead S. Nursing diagnosis, outcomes and interventions - NA NDA, NOC and NIC linkages. Saint Louis: M osby; 2001.

2. McCloskey JC, Bulechek GM. Classificação das

Cogitare Enferm 2007 jan/mar; 12(1):9-19 
Intervenções de Enfermagem (NIC). São Paulo: A rtmed; 2004.

3. North A merican Nursing Diagnosis A ssociation (NA NDA ). Diagnósticos de Enfermagem da NA NDA: definições e classificação 2001-2002. Porto Alegre: A rtmed; 2002.

4. Kim M J, Larson J L. Ineffective airway clearance and Ineffective breathing patterns. Nurs Clin North A m 1987; 22(1):125-34.

5. Carlson-Catalano J, L unney M, Paradiso C, B runo J, Luise BK, M artin T, M assoni M, Pachter S. Clinical validation of ineffective breathing pattern, ineffective ai rw ay clearance, and impaired gas exchange. Image J Nurs Sch 1998; 30(3):243-48.

6. Jarvis C. Exame físico e avaliação de saúde. Rio de Janeiro: Guanabara K oogan; 2002.

7. Pillitteri A. Child health nursing: care of the child and family. Philadelphia: Lippincott; 1999.

8. Wong DL. Enfermagem pediátrica: elementos essenciais à intervenção efetiva. Rio de Janeiro: Guanabara K oogan; 1999.

9. Shekleton $\mathrm{ME}, \mathrm{N}$ ield $\mathrm{M}$. Ineffective airway clearance related to artificial airway. Nurs Clin North A $m$ 1987; 22(1):167-78.

10. Consel ho Regional de Enfermagem do Estado de São Paulo (COREN-SP). Decreto no 94.406 , de 08 de junho de 1987. Regula a L ei $n^{\circ} 7498$ de 25 de junho de 1986, que dispõe sobre o exercício da enfermagem [acesso em 2007 Fev 24]. Disponível em: http://corensp.org.br/ 072005

11. Yin RK. Estudo de caso - planejamento e metodos. Porto A legre: B ookman; 2004.

12. Coenen $A$, Ryan $P$, Sutton $J$. M apping nursing interventions from a hospital information system to the N ursing Interventions Classification (NIC). N urs Diagn 1997; 8(4):145-51.

13. M artins I, Gutiérrez M GR. Intervenções de enfermagem para o diagnóstico de enfermagem Desobstrução ineficaz de vias aéreas. Acta Paul Enferm 2005; 18(2):143-9.

14. Guimarães HCQCP, B arros A LB L . Controlar líquidos: uma intervenção de enfermagem para o paciente com excesso de volume de líquidos. Rev L atino-am Enferm 2003; 11(6):734-41.

15. Hanley MV, Tyler M L. Ineffective airway clearance related to airway infection. N urs Clin N orth A m 1987; 22(1):135-50.

16. Shekleton M E, Nield M . Ineffective airway clearance related to artificial airway. N urs Clin N orth A m 1987; 22(1):167-78.

17. Potter PA, Perry A G. Fundamentos de enfermagem. Rio de J aneiro: Elsevier; 2005.

18. Carpenito $L J$. Diagnósticos de enfermagem. A plicação à prática clínica. Porto A legre: A rtmed; 2006.

19. Smeltzer SC, B areBG. Tratado de enfermagem médicocirúrgica. Rio de J aneiro: Guanabara K oogan; 2005.

20. Doenges M E, M oorhouse M F, Geissler A C. Planos de cuidado de enfermagem: orientações para o cuidado individualizado do paciente. Rio de J aneiro: Guanabara Koogan; 2003.

21. Payne J. The nursing interventions classification: a language to define nursing. Oncol N urs Forum 2000; 27(1):99-103.

22. Barros $A L B L, F a k i h ~ F T, M i c h e l ~ J L M . O$ uso do computador como ferramenta para a implementação do processo de enfermagem - a experiência do hospital São Paulo/UNIFESP. Rev Bras Enferm 2002; 55(6):714-19. 